\title{
DYNAMICS OF PROFIT-SHARING GAMES
}

\author{
John Augustine, ${ }^{1}$ Ning Chen, ${ }^{2}$ Edith Elkind, ${ }^{2,3}$ Angelo Fanelli, ${ }^{2,4}$ \\ Nick Gravin, ${ }^{2,5}$ and Dmitry Shiryaev ${ }^{2}$ \\ ${ }^{1}$ Department of Computer Science and Engineering, Indian Institute of Technology \\ Madras, Chennai, India \\ ${ }^{2}$ Division of Mathematical Sciences, School of Physical and Mathematical Sciences, \\ Nanyang Technological University, Singapore, Singapore \\ ${ }^{3}$ Department of Computer Science, Oxford University, Oxford, UK \\ ${ }^{4}$ Centre National de la Recherche Scientifique, Laboratoire CREM (UMR 6211), \\ Université de Caen Basse Normandie, Caen, France \\ ${ }^{5}$ Microsoft Research New England, Cambridge, Massachusetts, USA
}

\begin{abstract}
An important task in the analysis of multiagent systems is to understand how groups of selfish players can form coalitions, i.e., work together in teams. In this paper, we study the dynamics of coalition formation under bounded rationality. We consider settings whereby each team's profit is given by a submodular function and propose three profit-sharing schemes, each of which is based on the concept of marginal utility. The agents are assumed to be myopic, i.e., they keep changing teams as long as they can increase their payoff by doing so. We study the properties (such as closeness to Nash equilibrium or total profit) of the states that result after a polynomial number of such moves, and prove bounds on the price of anarchy and the price of stability of the corresponding games.
\end{abstract}

\section{INTRODUCTION}

Cooperation and collaborative task execution are fundamentally important both for human societies and for multiagent systems. Indeed, it is often the case that certain tasks are too complicated or resource-consuming to be executed by a single agent, and a collective effort is needed. Such settings are usually modeled using the framework of cooperative games, which specify the amount of payoff that each subset of agents can achieve: when the game is played, the agents split into teams (coalitions), and the payoff of each team is divided among its members.

The standard framework of cooperative game theory is static, i.e., it does not explain how the players arrive at a particular set of teams and a payoff distribution. However, understanding the dynamics of coalition formation is an obviously important issue from the practical perspective, and there is an active stream of research that studies bargaining and coalition formation in cooperative games (see, e.g., [11, 31, 33, 41]). Most of this research assumes that the agents are fully rational, i.e., that they can predict

Address correspondence to Angelo Fanelli, Centre National de la Recherche Scientifique, Laboratoire CREM (UMR 6211), Université de Caen Basse Normandie, 14032 Caen, France. E-mail: angelo.fanelli@ unicaen.fr 
the consequences of their actions and maximize their (expected) utility based on those predictions.

However, full rationality is a strong assumption that is unlikely to hold in many real-life scenarios: first, the agents may not have the computational resources to deduce their optimal strategies, and second, they may not be sophisticated enough to do so, or lack information about other players. Such agents may simply respond to their current environment without worrying about the subsequent reaction of other agents; such behavior is said to be myopic. Coalition formation by computationally limited agents has been studied by a number of researchers on multiagent systems, starting with [38] and [35]. However, myopic behavior in coalition formation has received relatively little attention in the literature (for some exceptions, see $[3,10,18]$ ). In contrast, myopic dynamics of noncooperative games is the subject of a growing body of research (see, e.g., $[6,20,23])$.

In this paper, we merge these streams of research and apply techniques developed in the context of analyzing the dynamics of noncooperative games to coalition-formation settings. In doing so, we depart from the standard model of games with transferable utility, which allows the players in a team to share the payoff arbitrarily: indeed, such flexibility will necessitate a complicated negotiation process whenever a player wants to switch teams. Instead, we consider three payoff models that are based on the concept of marginal utility, i.e., the contribution that the player makes to his current team. Each of the payoff schemes, when combined with a cooperative game, induces a noncooperative game whose dynamics can then be studied using the rich set of tools developed for such games in recent years.

We will now describe our payment schemes in more detail. We assume that we are given a cooperative game in which the values of the teams are given by a submodular function; the submodularity property means that a player is more useful when he joins a smaller team, and this plays an important role in our analysis. In our first scheme, the payment to each agent is given by his marginal utility for his current team; by submodularity, the total payment to the team members never exceeds the team's value. This payment scheme rewards each agent according to the value he creates; we will therefore call these games fair-value games.

Our second scheme takes into account the history of the interaction: we keep track of the order in which the players have joined their teams and pay each agent his marginal contribution to the coalition formed by the players who joined his current team before him. This ensures that the entire payoff of each team is fully distributed among its members. Moreover, due to the submodularity property, a player's payoff never goes down as long as he stays with the same team, even if some of his predecessors leave. This payoff scheme is somewhat reminiscent of the reward schemes employed in industries with strong labor unions; we will therefore refer to these games as labor union games. Our third scheme can be viewed as a hybrid of the first two: it distributes the team's payoff according to the players' Shapley values, i.e., it pays each player his expected marginal contribution to a coalition formed by his predecessors when players are reordered randomly; the resulting games are called Shapley games.

\subsection{Our Contributions}

We study the equilibria and dynamics of the three games described above. We are interested in the properties of the states that can be reached by natural dynamics in a 
polynomial number of steps, in particular, whether such states are (close to) Nash equilibria and whether they result in high total productivity, which is equal to the sum of the teams' values (note that in fair-value games, the latter quantity may differ from the social welfare, which is the sum of players' payoffs).

We first show that all our games are potential games and hence admit a Nash equilibrium in pure strategies. We then argue that for each of our games, the price of anarchy is bounded by 2 . For the first two classes of games, we can also bound their $\alpha$-price of anarchy, i.e., the ratio between the total profit of the optimal coalition structure and that of the worst $\alpha$-Nash equilibrium, with a bound of $2+\alpha$. We also provide bounds on the price of stability for all three games. Further, for the first two classes of games, we show that the basic Nash dynamics converges in polynomial time to an approximately optimal state, where the approximation ratio is arbitrarily close to the price of anarchy; these results extend to basic $\alpha$-Nash dynamics and $\alpha$-price of anarchy. To obtain these results, we observe that both the fair-value games and the labor union games can be viewed as variants of $\beta$-nice games introduced in [6] and prove general convergence results for such games, which may be of independent interest. We then show that labor union games have additional desirable properties: in such games, the $\alpha$-Nash dynamics quickly converges to an $\alpha$-Nash equilibrium; also, if we start with the state in which each player is unaffiliated, the Nash dynamics converges to a Nash equilibrium after each player gets a chance to move.

The rest of the paper is organized as follows. After a brief overview of related work, we provide the required preliminaries in Section 2 Section 3 deals with $\beta$-nice games and lays the groundwork for the technical results in the next section. Then, in Section 4, we describe our three classes of games and present our results for these games. Section 5 explains the relationship between our games and the well-studied cut games. Section 6 presents our conclusions and directions for future work.

\subsection{Related Work}

The games studied in this paper belong to the class of potential games, introduced in [32]. In such games, every sequence of improvements by players converges to a pure Nash equilibrium. However, the number of steps can be exponential in the description of the game. The complexity of computing an (approximate) Nash equilibrium in various subclasses of potential games such as congestion games [34], cut games [36], or party affiliation games [20] has received considerable attention in recent years [8, 15, 20, 26, 39, 40]. A related issue is how long it takes for some form of best-response dynamics to reach an equilibrium $[2,6,14,25,30,39]$. Even if a Nash equilibrium cannot be reached quickly, a state reached after a polynomial number of steps may still have high social welfare; this question is studied, for example, in [15, 22, 23].

Profit-sharing games can be placed within the framework of distributed welfare games, which were recently (and independently) introduced in [29]. A distributed welfare game is a resource allocation game, whereby each player can select a subset of resources, each of which generates welfare, which may depend on the subset of players who chose this resource, and the welfare generated at each resource is distributed among its users. Marden and Wierman propose four distribution rules for such games, including a rule that is based on players' marginal contributions and a rule that is based on the Shapley value; for the case in which each player can choose at most one resource, the resulting games coincide with, respectively, fair-value games and Shapley games. Marden and Wierman prove that 
under certain conditions (including submodularity), these games are potential games and their price of anarchy is bounded by 2 ; however, they do not consider dynamics of these games or approximate Nash equilibria.

While the focus of our work is on profit-sharing games, similar issues have been studied extensively in the context of cost-sharing games. In particular, there is a number of papers that deal with the design of cost-sharing mechanisms in congestion settings $[4,12,13,21,27]$, or scheduling rules in machine-scheduling settings $[1,7,9,16])$; just as in our model, the goal is to design the rules for sharing a resource that guarantee the existence of Nash equilibria and good price of anarchy and stability.

The recent paper [24] studies the dynamics of a class of cooperative games known as additively separable hedonic games, where the focus is on the complexity of computing stable outcomes. While the class of all cooperative games with submodular utility functions considered in this paper is considerably broader than that of additively separable games, the paper [24] also studies notions of stability not considered here.

\section{PRELIMINARIES}

\subsection{Noncooperative Games}

A noncooperative game is defined by a tuple $\mathcal{G}=\left(N,\left(\Sigma_{i}\right)_{i \in N},\left(u_{i}\right)_{i \in N}\right)$, where $N=\{1,2, \ldots, n\}$ is the set of players, $\Sigma_{i}$ is the set of (pure) strategies of player $i$, and $u_{i}: \times_{i \in N} \Sigma_{i} \rightarrow \mathbb{R}^{+} \cup\{0\}$ is the payoff function of player $i$.

Let $\Sigma=\times_{i \in N} \Sigma_{i}$ be the strategy profile set or state set of the game, and let $S=$ $\left(s_{1}, s_{2}, \ldots, s_{n}\right) \in \Sigma$ be a generic state in which each player $i$ chooses strategy $s_{i} \in \Sigma_{i}$. Given a strategy profile $S=\left(s_{1}, s_{2}, \ldots, s_{n}\right)$ and a strategy $s_{i}^{\prime} \in \Sigma_{i}$, let $\left(S_{-i}, s_{i}^{\prime}\right)$ be the strategy profile obtained from $S$ by changing the strategy of player $i$ from $s_{i}$ to $s_{i}^{\prime}$, i.e., $\left(S_{-i}, s_{i}^{\prime}\right)=\left(s_{1}, s_{2}, \ldots, s_{i-1}, s_{i}^{\prime}, s_{i+1}, \ldots, s_{n}\right)$.

\subsection{Nash Equilibria and Dynamics}

Given a strategy profile $S=\left(s_{1}, s_{2}, \ldots, s_{n}\right)$, a strategy $s_{i}^{\prime} \in \Sigma_{i}$ is an improvement move for player $i$ if $u_{i}\left(S_{-i}, s_{i}^{\prime}\right)>u_{i}(S)$; further, $s_{i}^{\prime}$ is called an $\alpha$-improvement move for $i$ if $u_{i}\left(S_{-i}, s_{i}^{\prime}\right)>(1+\alpha) u_{i}(S)$, where $\alpha>0$. A strategy $s_{i}^{b} \in \Sigma_{i}$ is a best response for player $i$ in state $S$ if it yields the maximum possible payoff given the strategy choices of the other players, i.e., $u_{i}\left(S_{-i}, s_{i}^{b}\right) \geq u_{i}\left(S_{-i}, s_{i}^{\prime}\right)$ for every $s_{i}^{\prime} \in \Sigma_{i}$. An $\alpha$-best response move is both an $\alpha$-improvement and a best response move.

A (pure) Nash equilibrium is a strategy profile in which every player plays her best response. Formally, $S=\left(s_{1}, s_{2}, \ldots, s_{n}\right)$ is a Nash equilibrium if for every $i \in N$ and strategy $s_{i}^{\prime} \in \Sigma_{i}$, we have $u_{i}(S) \geq u_{i}\left(S_{-i}, s_{i}^{\prime}\right)$. We denote the set of all (pure) Nash equilibria of a game $\mathcal{G}$ by $\mathcal{N} \mathcal{E}(\mathcal{G})$.

A profile $S=\left(s_{1}, \ldots, s_{n}\right)$ is called an $\alpha$-Nash equilibrium if no player can improve his payoff by more than a factor of $(1+\alpha)$ by deviating, i.e., $(1+\alpha) u_{i}(S) \geq u_{i}\left(S_{-i}, s_{i}^{\prime}\right)$ for every $i \in N$ and $u_{i}^{\prime} \in \Sigma_{i}$. The set of all $\alpha$-Nash equilibria of $\mathcal{G}$ is denoted by $\mathcal{N} \mathcal{E}^{\alpha}(\mathcal{G})$

In a strong Nash equilibrium, no group of players can improve their payoffs by deviating, i.e., $S=\left(s_{1}, \ldots, s_{n}\right)$ is a strong Nash equilibrium if for every $I \subseteq N$ and 
strategy vector $S^{\prime}=\left(s_{1}^{\prime}, \ldots, s_{n}^{\prime}\right)$ such that $s_{i}^{\prime}=s_{i}$ for $i \in N \backslash I$, if $u_{i}\left(S^{\prime}\right)>u_{i}(S)$ for some $i \in I$, then $u_{j}\left(S^{\prime}\right)<u_{j}(S)$ for some $j \in I$.

Let $\Delta_{i}(S)$ be the improvement in a player's payoff if he performs his best response, i.e., $\Delta_{i}(S)=u_{i}\left(S_{-i}, s_{i}^{b}\right)-u_{i}(S)$, where $s_{i}^{b}$ is the best response of player $i$ in state $S$. For every $Z \subseteq N$, let $\Delta_{Z}(S)=\sum_{i \in Z} \Delta_{i}(S)$, and let $\Delta(S)=\Delta_{N}(S)$.

A Nash dynamics (respectively $\alpha$-Nash dynamics) is any sequence of best response (respectively $\alpha$-best response) moves. A basic Nash dynamics (respectively basic $\alpha$-Nash dynamics) is any Nash dynamics (respectively $\alpha$-Nash dynamics) such that at each state $S$, the player $i$ that makes a move has the maximum absolute improvement, i.e., $i \in$ $\arg \max _{j \in N} \Delta_{j}(S)$.

\subsection{Price of Anarchy}

Given a game $\mathcal{G}$ with a set of states $\Sigma$ and a function $f: \Sigma \rightarrow \mathbb{R}^{+} \cup\{0\}$, we write $\operatorname{OPT}_{f}(\mathcal{G})=\max _{S \in \Sigma} f(S)$. The price of anarchy $\operatorname{PoA}_{f}(\mathcal{G})$ and the price of stability $\operatorname{PoS}_{f}(\mathcal{G})$ of a game $\mathcal{G}$ with respect to a function $f$ are, respectively, the worst-case ratio and the best-case ratio between the value of $f$ in a Nash equilibrium and $\operatorname{OPT}_{f}(\mathcal{G})$, i.e.,

$$
\operatorname{PoA}_{f}(\mathcal{G})=\max _{S \in \mathcal{N} \mathcal{E}(\mathcal{G})} \frac{\operatorname{OPT}_{f}(\mathcal{G})}{f(S)}, \quad \operatorname{PoS}_{f}(\mathcal{G})=\min _{S \in \mathcal{N} \mathcal{E}(\mathcal{G})} \frac{\mathrm{OPT}_{f}(\mathcal{G})}{f(S)} .
$$

The strong price of anarchy and the strong price of stability are defined similarly; the only difference is that the maximum (respectively minimum) is taken over all strong Nash equilibria. Further, the $\alpha$-price of anarchy $\operatorname{PoA}_{f}^{\alpha}(\mathcal{G})$ of a game $\mathcal{G}$ with respect to $f$ is defined as

$$
\operatorname{PoA}_{f}^{\alpha}(\mathcal{G})=\max _{S \in \mathcal{N} \mathcal{E}^{\alpha}(\mathcal{G})} \frac{\mathrm{OPT}_{f}(\mathcal{G})}{f(S)} ;
$$

the $\alpha$-price of stability $\operatorname{PoS}_{f}^{\alpha}(\mathcal{G})$ can be defined similarly. Originally, these notions were defined with respect to the social welfare function, i.e., $f=\sum_{i \in N} u_{i}(S)$. However, we give a more general definition, since in the setting of this paper, it is natural to use a different function $f$. We omit the index $f$ when the function $f$ is clear from the context.

\subsection{Potential Games}

A noncooperative game $\mathcal{G}$ is called a potential game if there is a function $\Phi: \Sigma \rightarrow$ $\mathbb{N}$ such that for every state $S$ and improvement move $s_{i}^{\prime}$ of a player $i$ in $S$, we have $\Phi\left(S_{-i}, s_{i}^{\prime}\right)-\Phi(S)>0$; the function $\Phi$ is called the potential function of $\mathcal{G}$. The game $\mathcal{G}$ is called an exact potential game if we have $\Phi\left(S_{-i}, s_{i}^{\prime}\right)-\Phi(S)=u_{i}\left(S_{-i}, s_{i}^{\prime}\right)-u_{i}(S)$. It is known that every potential game has a pure Nash equilibrium [32, 34].

\subsection{Cooperative Games}

A cooperative game $G=(N, v)$ is given by a set of players $N$ and a characteristic function $v: 2^{N} \rightarrow \mathbb{R}^{+} \cup\{0\}$ that for each set $I \subseteq N$ specifies the profit that the players in $I$ can earn by working together. We assume that $v(\varnothing)=0$. A coalition structure over $N$ is a partition of players in $N$, i.e., a collection of sets $I_{1}, \ldots, I_{k}$ such that (i) $I_{i} \subseteq N$ for $i=1, \ldots, k$; (ii) $I_{i} \cap I_{j}=\varnothing$ for all $i<j \leq k$; and 
(iii) $\cup_{j=1}^{k} I_{j}=N$. A game $G=(N, v)$ is called monotone if $v$ is nondecreasing, i.e., $v(I) \leq v(J)$ for every $I \subset J \subseteq N$. Further, $v$ is called submodular if for every $I \subset J \subseteq N$ and $i \in N \backslash J$, we have $v(I \cup\{i\})-v(I) \geq v(J \cup\{i\})-v(J)$. Informally, if $v$ is submodular, a player is more useful when he joins a smaller coalition. We will make use of the following property of submodular functions.

Lemma 2.1. Let $f: 2^{V} \rightarrow \mathbb{R}$ be a submodular function. Then for every pair of sets $X, Y \subseteq V$ such that $X \cap Y=\varnothing$ and $X=\left\{x_{1}, x_{2}, \ldots, x_{k}\right\}$, one has $\sum_{j=1, \ldots, k}\left(f\left(Y \cup\left\{x_{j}\right\}\right)-f(Y)\right) \geq f(Y \cup X)-f(Y)$.

Proof. Since $f$ is a submodular function, for every $x_{j} \in X$, we have

$$
f\left(Y \cup\left\{x_{j}\right\}\right)-f(Y) \geq f\left(Y \cup\left\{x_{1}, x_{2}, \ldots, x_{j-1}, x_{j}\right\}\right)-f\left(Y \cup\left\{x_{1}, x_{2}, \ldots, x_{j-1}\right\}\right) .
$$

The lemma now follows by summing these inequalities over all $j=1, \ldots, k$.

\section{PERFECT $\beta$-NICE GAMES}

In this section, we define the class of perfect $\beta$-nice games (our definition is inspired by [6] but differs from the one given there) and prove a number of results for such games. Subsequently, we will show that many of the profit-sharing games considered in this paper belong to that class.

Definition 3.1. A potential game $\mathcal{G}$ with a potential function $\Phi$ is called perfect with respect to a function $f: \Sigma \rightarrow \mathbb{R}^{+} \cup\{0\}$ if for every state $S$, we have $f(S) \geq \sum_{i \in N} u_{i}(S)$, and moreover, for every improvement move $s_{i}^{\prime}$ of player $i$, we have

$$
f\left(S_{-i}, s_{i}^{\prime}\right)-f(S) \geq \Phi\left(S_{-i}, s_{i}^{\prime}\right)-\Phi(S) \geq u_{i}\left(S_{-i}, s_{i}^{\prime}\right)-u_{i}(S) .
$$

Also, a game $\mathcal{G}$ is called $\beta$-nice with respect to a function $f: \Sigma \rightarrow \mathbb{R}^{+} \cup\{0\}$ if for every state $S$, we have $\beta \cdot f(S)+\Delta(S) \geq \operatorname{OpT}_{f}(\mathcal{G})$ and $f(S) \geq \sum_{i \in N} u_{i}(S)$.

We can bound the price of anarchy of a $\beta$-nice game by $\beta$.

Lemma 3.2. For every $f: \Sigma \rightarrow \mathbb{R}+\cup\{0\}$ and game $\mathcal{G}$ that is $\beta$-nice with respect to $f$, we have $\operatorname{PoA}_{f}(\mathcal{G}) \leq \beta$.

Proof. The lemma follows by observing that for every Nash equilibrium $S$, we have $\Delta(S) \leq 0$

Lemma 3.2 can be extended to the $\alpha$-price of anarchy for every $\alpha \geq 0$.

Lemma 3.3. For every $f: \Sigma \rightarrow \mathbb{R}^{+} \cup\{0\}, \alpha \geq 0$, and game $\mathcal{G}$ that is $\beta$-nice with respect to $f$, we have $\operatorname{PoA}_{f}^{\alpha}(\mathcal{G}) \leq \alpha+\beta$.

Proof. For every $\alpha$-Nash equilibrium $S$, we have $\Delta(S) \leq \alpha \sum_{i \in N} u_{i}(S) \leq \alpha f(S)$.

We now state a technical lemma that we shall use shortly in proving Theorem 3.5. 
Lemma 3.4. Consider a noncooperative game $\mathcal{G}$ and a function $f: \Sigma \rightarrow \mathbb{R}+\cup\{0\}$. For positive values of $\epsilon, a$, and $b$, any dynamics for which the increase in the value of $f$ at $a$ step leading from $S$ to $\bar{S}$ is at least $b-\frac{1}{a} f(S)$ converges from any initial state to a state $S^{F}$ with $f\left(S^{F}\right) \geq a b(1-\epsilon)$ in at most $\left\lceil a \ln \frac{1}{\epsilon}\right\rceil$ steps.

Proof. From the hypothesis, we have $f(\bar{S})-f(S) \geq b-\frac{1}{a} f(S)$. Let $h(S)=b-\frac{1}{a} f(S)$. Then

$$
h(S)-h(\bar{S})=\frac{1}{a}(f(\bar{S})-f(S)) \geq \frac{1}{a} h(S) .
$$

Hence

$$
h(\bar{S}) \leq\left(1-\frac{1}{a}\right) h(S)
$$

Consider a state $S^{F}$ that is reached by the dynamics starting from a state $S^{I}$ in $t$ steps. By recursively applying (31), we get

$$
h\left(S^{F}\right) \leq\left(1-\frac{1}{a}\right)^{t} h\left(S^{I}\right)
$$

By setting

$$
t=\left\lceil a \ln \frac{h\left(S^{I}\right)}{\epsilon b}\right\rceil \leq\left\lceil a \ln \frac{1}{\epsilon}\right\rceil
$$

in the previous inequality, we derive that $h\left(S^{F}\right) \leq \epsilon b$. Thus we obtain

$$
f\left(S^{F}\right)=a b\left(1-\frac{h\left(S^{F}\right)}{b}\right) \geq a b(1-\epsilon)
$$

The next theorem states that after a polynomial number of steps, for every perfect $\beta$-nice potential game, the basic Nash dynamics reaches a state whose relative quality (with respect to $f$ ) is close to the price of anarchy.

Theorem 3.5. Consider a function $f: \Sigma \rightarrow \mathbb{R}+\cup\{0\}$ and a game $\mathcal{G}$ that is perfect $\beta$-nice with respect to $f$. For every $\epsilon>0$, the basic Nash dynamics converges starting from any initial state to a state $S^{F}$ with

$$
f\left(S^{F}\right) \geq \frac{\operatorname{OPT}_{f}(\mathcal{G})}{\beta}(1-\epsilon)
$$

in at most $\left\lceil\frac{n}{\beta} \ln \frac{1}{\epsilon}\right\rceil$ steps.

Proof. Consider a generic state $S$ of the dynamics. Since $\mathcal{G}$ is $\beta$-nice, we have $\Delta(S) \geq$ $\mathrm{OPT}_{f}(\mathcal{G})-\beta \cdot f(S)$. Let $i$ be the player moving in state $S$, and let $\bar{S}$ be the state resulting from the move of player $i$. Since $i$ is the player with the maximum absolute improvement, 
we get

$$
f(\bar{S})-f(S) \geq \Phi(\bar{S})-\Phi(S) \geq \Delta_{i}(S) \geq \frac{\Delta(S)}{n} \geq \frac{\mathrm{OPT}_{f}(\mathcal{G})-\beta \cdot f(S)}{n} .
$$

The theorem now follows by applying Lemma 3.4 with $b=\operatorname{OPT}_{f}(\mathcal{G}) / n$ and $a=n / \beta$.

A convergence result similar to Theorem 3.5 can be obtained for basic $\alpha$-Nash dynamics.

Theorem 3.6. Consider a function $f: \Sigma \rightarrow \mathbb{R}^{+} \cup\{0\}$ and a game $\mathcal{G}$ that is perfect $\beta$-nice with respect to $f$. For every $\epsilon>0$ and $\alpha \geq 0$, the basic $\alpha$-Nash dynamics converges starting from any initial state to a state $S^{F}$ with

$$
f\left(S^{F}\right) \geq \frac{\operatorname{OPT}_{f}(\mathcal{G})}{\beta+\alpha}(1-\epsilon)
$$

in at most

$$
\left\lceil\frac{n}{\beta+\alpha} \ln \frac{1}{\epsilon}\right\rceil
$$

steps.

Proof. Let us consider a generic state $S=\left(s_{1}, \ldots, s_{n}\right)$ of the dynamics. Let $U \subseteq N$ be the subset of players that can perform an $\alpha$-best-response move, and let $E=N \backslash \bar{U}$. Note that no player $i \in E$ can improve his payoff by more than a factor of $1+\alpha$ by deviating from his current strategy, i.e., $\Delta_{E}(S) \leq \alpha \sum_{i \in E} u_{i}(S) \leq \alpha f(S)$. By definition of a perfect $\beta$-nice game, we have

$$
\Delta_{E}(S)+\Delta_{U}(S)=\Delta(S) \geq \mathrm{OPT}_{f}(\mathcal{G})-\beta \cdot f(S) .
$$

Let $i$ be the player moving in state $S$, and let $\bar{S}$ be the state resulting from the move of player $i \in U$. Since $i$ is the player with the maximum absolute improvement among the players in $U$, we get

$$
\begin{aligned}
f(\bar{S})-f(S) & \geq \Phi(\bar{S})-\Phi(S) \geq \Delta_{i}(S) \geq \frac{\Delta_{U}(S)}{|U|} \\
& \geq \frac{\operatorname{OPT}_{f}(\mathcal{G})-\beta \cdot f(S)-\Delta_{E}(S)}{n} \\
& \geq \frac{\operatorname{OPT}_{f}(\mathcal{G})-\beta \cdot f(S)-\alpha \cdot f(S)}{n} \\
& =\frac{\operatorname{OPT}_{f}(\mathcal{G})}{n}-\frac{\beta+\alpha}{n} f(S) .
\end{aligned}
$$

The theorem now follows by applying Lemma 3.4 with $b=\mathrm{OPT}_{f}(\mathcal{G}) / n$ and $a=n /(\beta+\alpha)$. 


\section{PROFIT-SHARING GAMES}

In this section, we study three noncooperative games that can be constructed from an arbitrary monotone cooperative game with a submodular utility function.

Each of our games can be described by a triple $\mathcal{G}=(N, v, M)$, where $(N, v)$ is a monotone cooperative game with a submodular utility function, $N=\{1, \ldots, n\}$, and $M=\{1, \ldots, m\}$ is a set of $m$ parties; we require $m \leq n$. All three games considered in this section model the setting whereby the players in $N$ form a coalition structure over $N$ that consists of $m$ coalitions. Thus, each player needs to choose exactly one party from $M$, i.e., for each $i \in N$ we have $\Sigma_{i}=M$. In some cases (see Section 4.2), we also allow players to be unaffiliated. To model this, we expand the set of strategies by setting $\Sigma_{i}=M \cup\{0\}$. Intuitively, the parties correspond to different companies, and the players correspond to the potential employees of these companies; we desire to assign employees to companies so as to maximize the total productivity.

In two of our games (see Section 4.1 and Section 4.3), a state of the game is completely described by the assignment of the players to the parties, i.e., we can write $S=\left(s_{1}, \ldots, s_{n}\right)$, where $s_{i} \in M$ for all $i \in N$. Alternatively, we can specify a state of the game by providing a partition of the set $N$ into $m$ components $Q_{1}, \ldots, Q_{m}$, where $Q_{j}$ is the set of all players that chose party $j$, i.e., we can write $S=\left(Q_{1}, \ldots, Q_{m}\right)$; we will use both forms of notation throughout the paper. In the game described in Section 4.2, the state of the game depends not only on which parties the players chose, but also on the order in which they joined the party; we postpone the formal description of this model till Section 4.2 In all three models, each player's payoff is based on the concept of marginal utility; however, in different models this idea is instantiated in different ways.

An important parameter of a state $S=\left(Q_{1}, \ldots, Q_{m}\right)$ in each of these games is its total profit $\operatorname{tp}(S)=\sum_{j \in M} v\left(Q_{j}\right)$. While for the games defined in Sections 4.2 and 4.3, the total profit coincides with the social welfare, for the game described in Section 4.1 this is not necessarily the case. Since we are interested in finding the most efficient partition of players into teams, we consider the total profit of a state a more relevant quantity than its social welfare. Therefore, in what follows, we will consider the price of anarchy and the price of stability with respect to the total profit, i.e., we have $\operatorname{Opt}(\mathcal{G})=\operatorname{Opt}_{\text {tp }}(\mathcal{G})$, $\operatorname{PoA}(\mathcal{G})=\operatorname{PoA}_{\mathrm{tp}}(\mathcal{G}), \operatorname{PoS}(\mathcal{G})=\operatorname{PoS}_{\mathrm{tp}}(\mathcal{G})$.

All of our results generalize to the setting in which each party $j \in M$ is associated with a different nondecreasing submodular profit function $v_{j}: 2^{N} \rightarrow \mathbb{R}+\cup\{0\}$; i.e., different companies possess different technologies, and therefore may have different levels of productivity. Formally, such a game is given by a tuple $\mathcal{G}=\left(N, v_{1}, \ldots, v_{m}, M\right)$, where $M=\{1, \ldots, m\}$, and for each $j \in M$, the function $v_{j}$ is a nondecreasing submodular function $v_{j}: 2^{N} \rightarrow \mathbb{R}^{+} \cup\{0\}$ that satisfies $v(\varnothing)=0$. In this case, the total profit function in a state $S=\left(Q_{1}, \ldots, Q_{m}\right)$ is given by $\operatorname{tp}(S)=\sum_{j \in M} v_{j}\left(Q_{j}\right)$. In what follows, we present our results for this more general setting.

\subsection{Fair-Value Games}

In our first model, the utility $u_{i}(S)$ of a player $i$ in a state $S=\left(Q_{1}, \ldots, Q_{m}\right)$ is given by $i$ 's marginal contribution to the coalition to which she belongs, i.e., if $i \in Q_{j}$, we set $u_{i}(S)=v_{j}(S)-v_{j}(S \backslash\{i\})$. Since this payment scheme rewards each player according to the value he creates, we will refer to such games as fair-value games. Observe that since 
the functions $v_{j}$ are assumed to be submodular, we have $\sum_{i \in Q_{j}} u_{i}(S) \leq v_{j}\left(Q_{j}\right)$ for all $j \in M$, i.e., the total payment to the employees of a company never exceeds the profit of the company. Moreover, it may be the case that the profit of a company is strictly greater than the amount it pays to its employees; we can think of the difference between the two quantities as the owner's or shareholders' value. Consequently, in these games the total profit of all parties may differ from the social welfare, as defined in Section 2

We will now argue that fair-value games have a number of desirable properties. In particular, every such game is a potential game, and therefore has a pure Nash equilibrium.

Theorem 4.1. Every fair-value game $\mathcal{G}$ is a perfect 2-nice exact potential game with respect to the total profit function.

Proof. It is easy to see that $\mathcal{G}$ is an exact potential game, whose potential function is given by the total profit. In order to prove the theorem, we need to show that for each state $S$, we have $2 \cdot \operatorname{tp}(S)+\Delta(S) \geq \operatorname{Opt}(\mathcal{G})$. Consider any state $S=\left(s_{1}, s_{2}, \ldots, s_{n}\right)$, and let $S^{\prime}=\left(s_{1}^{\prime}, s_{2}^{\prime}, \ldots, s_{n}^{\prime}\right)$ be the state of best responses to $S$, that is, let $s_{i}^{\prime}$ be the best response of player $i$ in state $S$. Moreover, let $S^{*}=\left(s_{1}^{*}, s_{2}^{*}, \ldots, s_{n}^{*}\right)$ be a state that maximizes the total profit. Consider a party $k \in M$, and let

$$
Q_{k}=\left\{i \in N \mid s_{i}=k\right\}, \quad Q_{k}^{*}=\left\{i \in N \mid s_{i}^{*}=k\right\} .
$$

We obtain

$$
\begin{aligned}
\Delta_{Q_{k}^{*}}(S) & =\sum_{j \in Q_{k}^{*}}\left(u_{j}\left(S_{-j}, s_{j}^{\prime}\right)-u_{j}(S)\right) \\
& \geq \sum_{j \in Q_{k}^{*}}\left(u_{j}\left(S_{-j}, k\right)-u_{j}(S)\right) \\
& =\sum_{j \in Q_{k}^{*}} u_{j}\left(S_{-j}, k\right)-\sum_{j \in Q_{k}^{*}} u_{j}(S) \\
& =\sum_{j \in Q_{k}^{*} \backslash Q_{k}}\left(v_{k}\left(Q_{k} \cup\{j\}\right)-v_{k}\left(Q_{k}\right)\right)+\sum_{j \in Q_{k}^{*} \cap Q_{k}} u_{j}(S)-\sum_{j \in Q_{k}^{*}} u_{j}(S) \\
& \geq v_{k}\left(Q_{k} \cup\left(Q_{k}^{*} \backslash Q_{k}\right)\right)-v_{k}\left(Q_{k}\right)-\sum_{j \in Q_{k}^{*}} u_{j}(S) \\
\geq & v_{k}\left(Q_{k}^{*}\right)-v_{k}\left(Q_{k}\right)-\sum_{j \in Q_{k}^{*}} u_{j}(S),
\end{aligned}
$$

where (41) holds because for each player $j$, the improvement from selecting the best response $s_{j}^{\prime}$ is at least the improvement achieved by choosing the optimal strategy $s_{j}^{*}=k$; (42) follows from Lemma 2.1; and (43) holds because $v_{k}$ is nondecreasing.

Summing these inequalities over all parties $k$, we obtain

$$
\begin{aligned}
\Delta(S) & =\sum_{k \in M} \Delta_{Q_{k}^{*}}(S) \geq \sum_{k \in M} v_{k}\left(Q_{k}^{*}\right)-\sum_{k \in M} v_{k}\left(Q_{k}\right)-\sum_{k \in M} \sum_{j \in Q_{k}^{*}} u_{j}(S) \\
& =\operatorname{tp}\left(S^{*}\right)-\operatorname{tp}(S)-\sum_{j \in N} u_{j}(S) \geq \operatorname{OPT}(\mathcal{G})-2 \operatorname{tp}(S)
\end{aligned}
$$


where the last inequality follows from the fact that for every state $S$, we have $\sum_{j \in N} u_{j}(S) \leq$ $\operatorname{tp}(S)$.

Combining Theorem 4.1, Lemmas 3.2 and 3.3, and Theorems 3.5 and 3.6, we obtain the following corollaries.

Corollary 4.2. For every fair-value game $\mathcal{G}$ and every $\alpha \geq 0$, we have $\operatorname{PoA}^{\alpha}(\mathcal{G}) \leq 2+\alpha$. In particular, $\operatorname{PoA}(\mathcal{G}) \leq 2$.

Corollary 4.3. For every fair-value game $\mathcal{G}$ and every $\epsilon>0$, the basic Nash dynamics (respectively the basic $\alpha$-Nash dynamics) converges from any initial state to a state $S^{F}$ with total profit

$$
\operatorname{tp}\left(S^{F}\right) \geq \frac{\text { OРт }(\mathcal{G})}{2}(1-\epsilon)
$$

respectively

$$
\operatorname{tp}\left(S^{F}\right) \geq \frac{\operatorname{OPT}(\mathcal{G})}{2+\alpha}(1-\epsilon)
$$

in at most $\left\lceil\frac{n}{2} \ln \frac{1}{\epsilon}\right\rceil$ steps (respectively $\left\lceil\frac{n}{2+\alpha} \ln \frac{1}{\epsilon}\right\rceil$ steps).

Since every fair-value game is an exact potential game with the potential function given by the total profit, every profit-maximizing state is necessarily a Nash equilibrium. This implies the following proposition.

Proposition 4.4. For every fair-value game $\mathcal{G}$, we have $\operatorname{PoS}(\mathcal{G})=1$.

\subsection{Labor Union Games}

In fair-value games, the player's payoff depends only on his current marginal value to the enterprise, i.e., one's salary may go down as the company expands. However, in many real-life settings, this is not the case. For instance, in many industries, especially ones that are highly unionized, an employee that has spent many years working for the company typically receives a higher salary than a new hire with the same set of skills. Our second class of games, which we will refer to as labor union games, aims to model this type of setting. Specifically, in this class of games, we modify the notion of state so as to take into account the order in which the players have joined their respective parties; the payment to each player is then determined by his marginal utility for the coalition formed by his predecessors. The submodularity property guarantees than a player's payoff never goes down as long as he stays with the same party.

Formally, we allow the players in a labor union game $\mathcal{G}$ that corresponds to a tuple $\left(N, v_{1}, \ldots, v_{m}, M\right)$, to be unaffiliated, i.e., for each $i \in N$, we set $\Sigma_{i}=M \cup\{0\}$. If player $i$ plays strategy 0 , we set his payoff to be 0 irrespective of the other players' strategies. A state of $\mathcal{G}$ is given by a tuple $S=\left(P_{1}, \ldots, P_{m}\right)$, where $P_{j}$ is the sequence of players in party $j$, ordered according to their arrival time.

We denote by $\bar{P}_{j}$ the corresponding set of all players in party $j$. As before, the profit of party $j$ is given by the function $v_{j}$; note that the value of $v_{j}$ does not depend on the order in 
which the players join $j$. The payoff of each player, however, is dependent on her position in the affiliation order. Specifically, for a player $i$ in party $j$, let $P_{j}(i)$ be the set of players that appear in $P_{j}$ before $i$. Player $i$ 's payoff is then defined as $u_{i}(S)=v_{j}\left(P_{j}(i) \cup\{i\}\right)-v_{j}\left(P_{j}(i)\right)$.

We remark that technically speaking, labor union games are not noncooperative games. Rather, each state of a labor union game induces a noncooperative game as described above; after a player makes a move, the induced noncooperative game changes. Abusing terminology, we will say that a state $S$ of a labor union game $\mathcal{G}$ is a Nash equilibrium if for each player $i \in N$, staying with his current party is a best response in the induced game; all other notions that were defined for noncooperative games in Section 2, as well as the results in Section 3, can be extended to labor union games in a similar manner.

We now state two fundamental properties of our model.

Guaranteed payoff. Consider two players $i$ and $i^{\prime}$ in the sequence $P_{j}$. Suppose $i^{\prime}$ moves to another party. The payoff of player $i$ will not decrease. Indeed, if $i^{\prime}$ succeeds $i$ in the sequence $P_{j}$, then by definition, $i$ 's payoff is unchanged. If $i^{\prime}$ precedes $i$ in $P_{j}$, then since $v_{j}$ is nondecreasing and submodular, $i$ 's payoff will not decrease; it may, however, increase.

Full payoff distribution. The sum of the payoffs of players within a party $j$ is a telescopic sum that evaluates to $v_{j}\left(\bar{P}_{j}\right)$. Therefore, the total profit $\operatorname{tp}(S)=\sum_{j \in M} v_{j}\left(\bar{P}_{j}\right)$ in a state $S$ equals the social welfare in this state. In other words, in labor union games, the profit of each enterprise is distributed among its employees without creating any value for the owners/shareholders.

The guaranteed payoff property distinguishes the labor union games from the fairvalue games, in which a player who maintains his affiliation to a party might not be rewarded, but may rather see a reduction in his payoff as other players move to join his party. This, of course, may incentivize him to shift his affiliation as well, leading to a vicious cycle of moves. In contrast, in labor union games, a player is guaranteed that his payoff will not decrease if he maintains his affiliation to a party. This suggests that in labor union games, stability may be easier to achieve. In what follows, we will see that this is indeed the case.

We will first show that labor union games are perfect 2-nice with respect to the total profit (or equivalently, social welfare); this will allow us to apply the machinery developed in Section 3 Abusing notation, let $\Delta_{i}(S)$ denote the improvement in the payoff of player $i$ if he performs a best-response move from $S$, and let $\Delta(S)=\sum_{i \in N} \Delta_{i}(S)$.

Proposition 4.5. Every labor union game $\mathcal{G}$ is a perfect 2-nice game with respect to the total profit function.

Proof. It is easy to see that $\mathcal{G}$ is a potential game with potential function $\Phi(S)=\operatorname{tp}(S)$. Furthermore, for every player $i$, the increase in his payoff when he performs an improvement move does not exceed the change in the total profit. It remains to show that $2 \operatorname{tp}(S)+\Delta(S) \geq$ Oрт $(\mathcal{G})$ for every $S=\left(P_{1}, \ldots, P_{m}\right)$.

Let $S^{*}=\left(P_{1}^{*}, \ldots, P_{m}^{*}\right)$ be a state that maximizes the total profit. We have

$$
\begin{aligned}
v_{j}\left(\bar{P}_{j}^{*}\right) & \leq v_{j}\left(\bar{P}_{j} \cup \bar{P}_{j}^{*}\right)=v_{j}\left(\bar{P}_{j}\right)+v_{j}\left(\bar{P}_{j} \cup \bar{P}_{j}^{*}\right)-v_{j}\left(\bar{P}_{j}\right) \\
& \leq v_{j}\left(\bar{P}_{j}\right)+\sum_{i \in \bar{P}_{j}^{*} \backslash \bar{P}_{j}}\left(u_{i}(S)+\Delta_{i}(S)\right) .
\end{aligned}
$$


Summing over all parties, we obtain

$$
\begin{aligned}
\operatorname{OpT}(\mathcal{G}) & =\sum_{j \in M} v_{j}\left(\bar{P}_{j}^{*}\right) \leq \sum_{j \in M} v_{j}\left(\bar{P}_{j}\right)+\sum_{j \in M} \sum_{i \in \bar{P}_{j}^{*} \backslash \bar{P}_{j}} u_{i}(S)+\sum_{j \in M} \sum_{i \in \bar{P}_{j}^{*} \backslash \bar{P}_{j}} \Delta_{i}(S) \\
& \leq 2 \operatorname{tp}(S)+\Delta(S)
\end{aligned}
$$

completing the proof.

As in the case of fair-value games, Proposition 4.5 allows us to bound the price of anarchy of any labor union game, as well as the time it takes to converge to a state with a "good" total profit.

Corollary 4.6. For every labor union game $\mathcal{G}$ and every $\alpha \geq 0$, we have $\operatorname{PoA}^{\alpha}(\mathcal{G}) \leq 2+\alpha$. In particular, $\operatorname{PoA}(\mathcal{G}) \leq 2$.

Corollary 4.7. For every labor union game $\mathcal{G}$ and $\epsilon>0$, the basic Nash dynamics (respectively the basic $\alpha$-Nash dynamics) converges from any initial state to a state $S^{F}$ with total profit

$$
\operatorname{tp}\left(S^{F}\right) \geq \frac{\text { OРт }(\mathcal{G})}{2}(1-\epsilon)
$$

respectively,

$$
\operatorname{tp}\left(S^{F}\right) \geq \frac{\operatorname{OPT}(\mathcal{G})}{2+\alpha}(1-\epsilon)
$$

in at most $\left\lceil\frac{n}{2} \ln \frac{1}{\epsilon}\right\rceil$ steps (respectively $\left\lceil\frac{n}{2+\alpha} \ln \frac{1}{\epsilon}\right\rceil$ steps).

Let $S^{*}=\left(P_{1}^{*}, \ldots, P_{m}^{*}\right)$ be a state that maximizes the total profit in a game $\mathcal{G}$, and let $\operatorname{Opt}(\mathcal{G})=\operatorname{tp}\left(S^{*}\right)$. As in the case of fair-value games, it is not hard to see that $S^{*}$ is a Nash equilibrium, i.e., $\operatorname{PoS}(\mathcal{G})=1$. In fact, for labor union games, we can prove a stronger statement.

Proposition 4.8. In every labor union game $\mathcal{G}, S^{*}$ is a strong Nash equilibrium, i.e., the strong price of stability is 1.

Proof. Consider a deviating coalition $I \subseteq N$. By the guaranteed payoff property, the deviation does not lower the payoff of all players in $N \backslash I$ and increases the payoff of some of the deviators, without harming the rest of the deviators. Thus, the deviation must lead to a state whose total payoff exceeds that of $S^{*}$, a contradiction.

Furthermore, for labor union games, we can show that for certain dynamics and certain initial states, one can guarantee convergence to an $\alpha$-Nash equilibrium or even a Nash equilibrium.

Proposition 4.9. For every labor union game $\mathcal{G}=\left(N, v_{1}, \ldots, v_{m}, M\right)$ such that the payoff of every player is at least 1 , the $\alpha$-Nash dynamics starting from any state in which all 
players are affiliated with some party converges to an $\alpha$-Nash equilibrium in $O\left(\frac{n}{\alpha} \log W\right)$ steps, where $W$ is the maximum payoff that any player can achieve.

Proof. After each move in the $\alpha$-Nash dynamics, a player improves her payoff by a factor of $1+\alpha$, and the guaranteed payoff property ensures that payoffs of other players are unaffected. So, if a player starts with a payoff of at least 1 , she will reach a payoff of $W$ after $O\left(\frac{\log W}{\alpha}\right)$ steps. Therefore, in $O\left(\frac{n}{\alpha} \log W\right)$ steps, we are guaranteed to reach an $\alpha$-Nash equilibrium.

We remark that the assumption that the payoff of each player is at least 1 is not restrictive: we can rescale the profit functions so that for every nonempty set of players, the profit is always at least 1 .

Proposition 4.10. Suppose a labor union game $\mathcal{G}$ with $n$ players starts at a state in which every player is unaffiliated. Then in exactly $n$ steps of the Nash dynamics, the system will reach a Nash equilibrium.

Proof. The proof is by induction on the number of steps. The very first player who gets to move will pick the party that maximizes her payoff. Subsequently, she will never have an incentive to move, because no move will give her any improvement in her payoff. For the inductive step, suppose that $k-1$ steps have elapsed, and exactly $k-1$ players have moved once each and have reached their final destination with no incentive to move again. The player who moves at step $k$ chooses his best-response party. Since the profit functions are increasing and submodular, he cannot improve his payoff by moving to another party at a later step. Therefore, in $n$ steps, the system reaches a Nash equilibrium.

We conclude with an important open question. We have shown that for $\alpha>0$, the $\alpha$-Nash dynamics leads to an $\alpha$-Nash equilibrium in $O\left(\frac{n}{\alpha} \log W\right)$ steps. However, we do not know whether there exists a dynamics that converges to a Nash equilibrium in a number of steps that is a polynomial in $n$ and $\log W$.

\subsection{Shapley Games}

In our third class of games, which we call Shapley games, the players' payoffs are determined in a way that is inspired by the definition of the Shapley value [37]. As in fair-value games, a state of a Shapley game is fully described by the partition of the players into parties. Given a state $S=\left(Q_{1}, \ldots, Q_{m}\right)$ and a player $i \in Q_{j}$, we define player $i$ 's payoff as

$$
u_{i}(S)=\sum_{Q \subseteq Q_{j} \backslash\{i\}} \frac{|Q| !\left(\left|Q_{j}\right|-|Q|-1\right) !}{\left|Q_{j}\right| !}\left(v_{j}(Q \cup\{i\})-v_{j}(Q)\right) .
$$

Intuitively, the payment to each player can be viewed as his average payment in the labor union model, where the average is taken over all possible orderings of the players in the party. This immediately implies $\sum_{i \in Q_{j}} u_{i}(S)=v_{j}\left(Q_{j}\right)$. Thus, Shapley games share features with both the fair-value games and the labor union games. As in fair-value games, the order in which the players join the party is unimportant. Moreover, if all payoff functions are additive, i.e., we have $u_{i}(S \cup\{j\})-u_{i}(S)=u_{i}(\{j\})$ for every $i \in N$ and $S \subseteq N \backslash\{i\}$, 
then the respective Shapley game coincides with the fair-value game that corresponds to $\left(N, v_{1}, \ldots, v_{m}, M\right)$. On the other hand, as with labor union games, the entire profit of each party is distributed among its members.

We will first show that every Shapley game is an exact potential game and hence admits a Nash equilibrium in pure strategies.

Theorem 4.11. Every Shapley game $\mathcal{G}=\left(N, v_{1}, \ldots, v_{m}, M\right)$ is an exact potential game with potential function given by

$$
\Phi(S)=\sum_{j \in M} \sum_{Q \subseteq Q_{j}} \frac{(|Q|-1) !\left(\left|Q_{j}\right|-|Q|\right) !}{\left|Q_{j}\right| !} v_{j}(Q) .
$$

Proof. Suppose that in some state $S=\left(Q_{1}, \ldots, Q_{m}\right)$ of the game, a player $i$ that belongs to party 1 wants to switch to party 2 . Let $S^{\prime}$ be the state after player $i$ switches. Our goal is to show that $u_{i}\left(S^{\prime}\right)-u_{i}(S)=\Phi\left(S^{\prime}\right)-\Phi(S)$, so $\Phi$ is indeed a potential function of the game.

We can compute the utility of player $i$ in both states, taking into account that in state $S$, player $i$ belongs to party 1 with $\left|Q_{1}\right|$ members, but in state $S^{\prime}$, she belongs to party 2 with $\left|Q_{2}+1\right|$ members:

$$
\begin{aligned}
& u_{i}\left(S^{\prime}\right)=\sum_{Q \subseteq Q_{2}} \frac{|Q| !\left(\left|Q_{2}\right|-|Q|\right) !}{\left(\left|Q_{2}\right|+1\right) !}\left(v_{2}(Q \cup\{i\})-v_{2}(Q)\right), \\
& u_{i}(S)=\sum_{Q \subseteq Q_{1} \backslash\{i\}} \frac{|Q| !\left(\left|Q_{1}\right|-|Q|-1\right) !}{\left|Q_{1}\right| !}\left(v_{1}(Q \cup\{i\})-v_{1}(Q)\right) .
\end{aligned}
$$

The only parties whose composition changes as we move from state $S$ to state $S^{\prime}$ are party 1 and party 2 . Therefore, when computing the difference between $\Phi\left(S^{\prime}\right)$ and $\Phi(S)$, we can ignore all other parties:

$$
\begin{aligned}
\Phi\left(S^{\prime}\right) & -\Phi(S) \\
= & \sum_{Q \subseteq Q_{1} \backslash\{i\}} \frac{(|Q|-1) !\left(\left|Q_{1}\right|-1-|Q|\right) !}{\left(\left|Q_{1}\right|-1\right) !} v_{1}(Q) \\
& +\sum_{Q \subseteq Q_{2} \cup\{i\}} \frac{(|Q|-1) !\left(\left|Q_{2}\right|+1-|Q|\right) !}{\left(\left|Q_{2}\right|+1\right) !} v_{2}(Q) \\
& -\sum_{Q \subseteq Q_{1}} \frac{(|Q|-1) !\left(\left|Q_{1}\right|-|Q|\right) !}{\left|Q_{1}\right| !} v_{1}(Q) \\
& -\sum_{Q \subseteq Q_{2}} \frac{(|Q|-1) !\left(\left|Q_{2}\right|-|Q|\right) !}{\left|Q_{2}\right| !} v_{2}(Q) \\
= & \sum_{Q \subseteq Q_{2}}\left(\left(\frac{(|Q|-1) !\left(\left|Q_{2}\right|+1-|Q|\right) !}{\left(\left|Q_{2}\right|+1\right) !}-\frac{(|Q|-1) !\left(\left|Q_{2}\right|-|Q|\right) !}{\left|Q_{2}\right| !}\right) v_{2}(Q)\right. \\
& \left.+\frac{(|Q|) !\left(\left|Q_{2}\right|-|Q|\right) !}{\left(\left|Q_{2}\right|+1\right) !} v_{2}(Q \cup\{i\})\right)
\end{aligned}
$$




$$
\begin{aligned}
& +\sum_{Q \subseteq Q_{1} \backslash\{i\}}\left(\left(\frac{(|Q|-1) !\left(\left|Q_{1}\right|-1-|Q|\right) !}{\left(\left|Q_{1}\right|-1\right) !}-\frac{(|Q|-1) !\left(\left|Q_{1}\right|-|Q|\right) !}{\left|Q_{1}\right| !}\right) v_{1}(Q)\right. \\
& \left.-\frac{(|Q|) !\left(\left|Q_{1}\right|-|Q|-1\right) !}{\left|Q_{1}\right| !} v_{1}(Q \cup\{i\})\right) \\
& =\sum_{Q \subseteq Q_{2}} \frac{|Q| !\left(\left|Q_{2}\right|-|Q|\right) !}{\left(\left|Q_{2}\right|+1\right) !}\left(v_{2}(Q \cup\{i\})-v_{2}(Q)\right) \\
& -\sum_{Q \subseteq Q_{1} \backslash\{i\}} \frac{|Q| !\left(\left|Q_{1}\right|-|Q|-1\right) !}{\left|Q_{1}\right| !}\left(v_{1}(Q \cup\{i\})-v_{1}(Q)\right)=u_{i}\left(S^{\prime}\right)-u_{i}(S),
\end{aligned}
$$

which completes the proof.

Just as in other profit-sharing games, the price of anarchy in Shapley games is bounded by 2 .

Theorem 4.12. In every Shapley game $\mathcal{G}=\left(N, v_{1}, \ldots, v_{m}, M\right)$ with $|N|=n$, we have $\operatorname{PoA}(\mathcal{G}) \leq 2-\frac{1}{n}$

Proof. Let $S=\left(Q_{1}, \ldots, Q_{m}\right)$ be a Nash equilibrium state, and suppose that $S^{*}=$ $\left(Q_{1}^{*}, \ldots, Q_{m}^{*}\right)$ is a state in which the maximum total profit is achieved. It suffices to show that

$$
\left(1-\frac{1}{n}\right) \operatorname{tp}(S)+\operatorname{tp}(S) \geq \operatorname{tp}\left(S^{*}\right)
$$

Observe first that if $\left|Q_{j}\right|=n$ for some $j \in M$, then $S$ is an optimal state. Indeed, if $S$ is not optimal, then by the total payoff distribution property, there exist a party $k \in M$ and a player $i \in Q_{k}^{*}$ such that $u_{i}\left(S^{*}\right)>u_{i}(S)$. If player $i$ switches to party $k$, which currently has no members, then by the submodularity property, his payoff will be at least $u_{i}\left(S^{*}\right)$, a contradiction to $S$ being a Nash equilibrium state. Therefore, from now on, we assume that $\left|Q_{j}\right|<n$ for all $j \in M$.

We now have

$$
\operatorname{tp}(S)=\sum_{i \in N} u_{i}(S)=\sum_{j \in M} \sum_{i \in Q_{j}^{*}} u_{i}(S) .
$$

For every $j \in M$ and $i \in Q_{j}^{*}$, we can derive a lower bound on $u_{i}(S)$. There are two cases to be considered.

(1) If $i \in Q_{j}$, we have

$$
\begin{aligned}
u_{i}(S) & =\sum_{Q \subseteq Q_{j} \backslash\{i\}} \frac{|Q| !\left(\left|Q_{j}\right|-|Q|-1\right) !}{\left|Q_{j}\right| !}\left(v_{j}(Q \cup\{i\})-v_{j}(Q)\right) \\
& >\sum_{Q \subset Q_{j} \backslash\{i\}} \frac{|Q| !\left(\left|Q_{j}\right|-|Q|\right) !}{\left(\left|Q_{j}\right|+1\right) !}\left(v_{j}(Q \cup\{i\})-v_{j}(Q)\right) .
\end{aligned}
$$


(2) If $i \notin Q_{j}$, we have

$$
u_{i}(S) \geq \sum_{Q \subseteq Q_{j}} \frac{|Q| !\left(\left|Q_{j}\right|-|Q|\right) !}{\left(\left|Q_{j}\right|+1\right) !}\left(v_{j}(Q \cup\{i\})-v_{j}(Q)\right),
$$

since $S$ is a Nash equilibrium, and hence player $i$ cannot increase his utility by switching to party $j$.

Changing the order of summation, by Lemma 2.1, we have

$$
\operatorname{tp}(S) \geq \sum_{j \in M} \sum_{Q \subseteq Q_{j}} \frac{|Q| !\left(\left|Q_{j}\right|-|Q|\right) !}{\left(\left|Q_{j}\right|+1\right) !}\left(v_{j}\left(Q \cup Q_{j}^{*}\right)-v_{j}(Q)\right) .
$$

Set $q=\left|Q_{j}\right|$. We have

$$
\begin{aligned}
\sum_{Q \subseteq Q_{j}} \frac{|Q| !\left(\left|Q_{j}\right|-|Q|\right) !}{\left(\left|Q_{j}\right|+1\right) !} & =\sum_{i=0}^{q} \sum_{Q \subseteq Q_{j},|Q|=i} \frac{|Q| !\left(\left|Q_{j}\right|-|Q|\right) !}{\left(\left|Q_{j}\right|+1\right) !}=\sum_{i=0}^{q}\left(\begin{array}{c}
q \\
i
\end{array}\right) \frac{i !(q-i) !}{(q+1) !} \\
& =\sum_{i=0}^{q} \frac{1}{q+1}=1 .
\end{aligned}
$$

This identity can also be derived by considering Shapley values in an additive game with $\left|Q_{j}\right|+1$ players. Further, we have $v_{j}\left(Q \cup Q_{j}^{*}\right) \geq v_{j}\left(Q_{j}^{*}\right)$. Thus

$$
\operatorname{tp}(S) \geq \sum_{j \in M} v_{j}\left(Q_{j}^{*}\right)-\sum_{j \in M} \sum_{Q \subseteq Q_{j}} \frac{|Q| !\left(\left|Q_{j}\right|-|Q|\right) !}{\left(\left|Q_{j}\right|+1\right) !} v_{j}(Q) .
$$

For every $Q \subseteq Q_{j}$, we have $v_{j}(Q) \leq v_{j}\left(Q_{j}\right)$, and moreover, $v_{j}(\varnothing)=0$. Recall also that we assume that $\left|Q_{j}\right|<n$ for all $j \in M$. Thus we can bound the negative term on the right-hand side of (44) as

$$
\begin{aligned}
\sum_{j \in M} \sum_{\substack{Q \subseteq Q_{j} \\
Q \neq \varnothing}} \frac{|Q| !\left(\left|Q_{j}\right|-|Q|\right) !}{\left(\left|Q_{j}\right|+1\right) !} v_{j}\left(Q_{j}\right) & =\sum_{j \in M}\left(1-\frac{0 !\left(\left|Q_{j}\right|-0\right) !}{\left(\left|Q_{j}\right|+1\right) !}\right) v_{j}\left(Q_{j}\right) \\
& \leq\left(1-\frac{1}{n}\right) \operatorname{tp}(S)
\end{aligned}
$$

Combining (44) and (45), we obtain $\left(2-\frac{1}{n}\right) \operatorname{tp}(S) \geq \operatorname{tp}\left(S^{*}\right)$.

The following claim shows that the bound given in Theorem 4.12 is almost tight.

Proposition 4.13. For every $n \geq 3$, there exists a Shapley game $\mathcal{G}=\left(N, v_{1}, v_{2}, M\right)$ with $|N|=n$ and $|M|=2$ such that

$$
\operatorname{PoA}(\mathcal{G})=2-\frac{2}{n+1} \quad \text { and } \quad \operatorname{PoS}(\mathcal{G})=2-\frac{4}{n+1} .
$$


Proof. Let $v_{1}$ be an additive function given by

$$
v_{1}(\{1\})=\frac{1}{n}, \quad v_{1}(\{i\})=\frac{1}{n-1}
$$

for $i \geq 2$, and let $v_{2}(Q)=1$ for every $Q \neq \varnothing$.

The state $S^{*}=\left(Q_{1}^{*}, Q_{2}^{*}\right)$ with $Q_{1}^{*}=\{2, \ldots, n\}, Q_{2}^{*}=\{1\}$ has total profit ( $n-$ 1) $\frac{1}{n-1}+1=2$, which is the optimum in this game.

On the other hand, a state $S=\left(Q_{1}, Q_{2}\right)$ with $Q_{1}=\{1\}, Q_{2}=\{2, \ldots, n\}$ is a Nash equilibrium. Indeed, player 1 is paid $1 / n$ and will be paid the same amount if he switches parties, so he has no incentive to switch. All other players are paid $\frac{1}{n-1}$, and any of them who switch to the first party will be paid the same amount. Therefore, none of them has an incentive to switch either. The total profit in state $S$ is $1+\frac{1}{n}$. There is no Nash equilibrium with a smaller total profit, because in any Nash equilibrium state there are players in both parties, and hence the total profit is at least $\frac{1}{n}+1$. Thus,

$$
\operatorname{PoA}(\mathcal{G})=\frac{2}{1+1 / n}=2-\frac{2}{n+1} .
$$

In every Nash equilibrium, party 2 contains at least $n-2$ players. Hence, the total profit in a Nash equilibrium is at most $\frac{2}{n-1}+1$. This profit is achieved in, e.g., state $S^{\prime}=\left(Q_{1}^{\prime}, Q_{2}^{\prime}\right)$ with $Q_{1}^{\prime}=\{n-1, n\}, Q_{2}^{\prime}=\{1, \ldots, n-2\}$. Therefore,

$$
\operatorname{PoS}(\mathcal{G})=\frac{2}{1+2 /(n-1)}=2-\frac{4}{n+1},
$$

which completes the proof.

\section{CUT GAMES AND PROFIT-SHARING GAMES}

We will now describe a family of succinctly representable profit-sharing games that can be described in terms of undirected weighted graphs. It turns out that while two wellstudied classes of games on such graphs do not induce profit-sharing games, a "hybrid" approach does. We then explain how to compute players' payoffs in the resulting profitsharing games.

In the classic cut games [15, 20,36], players are the vertices of a weighted graph $G=(N, E)$. The state of the game is a partition of players into two parties, and the payoff of each player is the sum of the weights of cut edges that are incident on him. A cut game naturally corresponds to a coalitional game with the set of players $N$, where the value of a coalition $S \subseteq N$ equals the weight of the cut induced by $S$ and $N \backslash S$. However, this game is not monotone, so it does not induce a profit-sharing game, as defined in Section 4

In induced subgraph games [17], the value of a coalition $S$ equals the total weight of all edges that have both endpoints in $S$; while these games are monotone, their utility function is not necessarily submodular.

Finally, consider a game in which the value of a coalition $S \subseteq N$ equals the total weight of all edges incident on vertices in $S$, i.e., both internal edges of $S$ (as in induced subgraph games) and the edges leaving $S$ (as in cut games). It is not hard to see that this game is monotone and has a submodular utility function, and hence induces a profit-sharing game as described in Section 4 We will now explain how to compute players' payoffs in the corresponding fair-value games, labor union games, and Shapley games, using Figure 1. In 


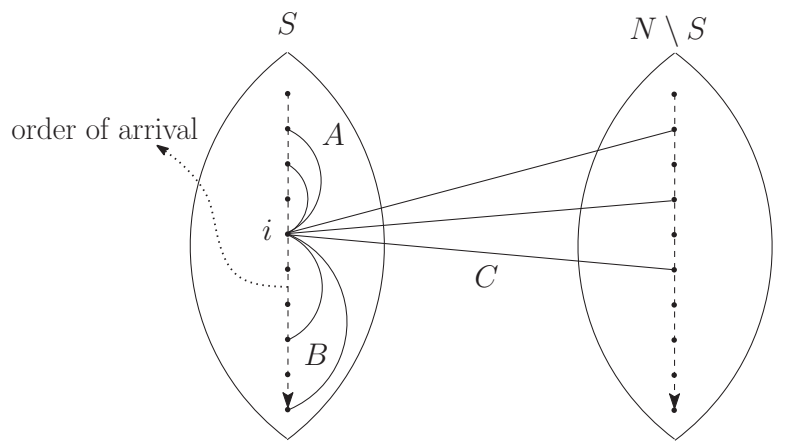

Figure 1 The set $N$ of players is partitioned into parties $S$ and $N \backslash S$. Consider a player $i$. Then $A$ denotes the total weight of edges incident on $i$ and connecting $i$ to a predecessor within the party, while $B$ denotes the total weight of edges incident on $i$ and connecting $i$ to a successor within the party. And $C$ is the total weight of the cut edges incident on $i$.

this figure, we are given a state of the game with two parties $S$ and $N \backslash S$; the players are listed from top to bottom in the order in which they (last) entered each party. (The order is relevant only in labor union games.) Here $A$ denotes the total weight of edges incident on $i$ that connect $i$ to a predecessor within the party, while $B$ denotes the total weight of edges incident on $i$ that connect $i$ to a successor within the party. And $C$ is the total weight of the cut edges incident on $i$. One can interpret an edge $e=\left(i, i^{\prime}\right)$ with weight $w(e)$ as a skill or resource of value proportional to $w(e)$ that both $i$ and $i^{\prime}$ possess.

Fair-value games. The payoff of $i$ (see Figure 1) is given by $\frac{1}{2}(A+B)+C$. Intuitively, the unique skills of a player are weighted more toward his payoff than his shared skills.

Labor union games. The payoff of $i$ is given by $B+C$. Intuitively, $i$ 's payoff reflects the unique skills that $i$ possessed when he joined the party. Players who share skills with $i$, but join after $i$, will not get any payoff for those shared skills.

Shapley games. One can show that $i$ 's payoff is given by $\frac{1}{2}(A+B)+C$, just as in fair-value games.

One can see that this interpretation easily extends to multiple parties and hyperedges. We also note that many of the notions that we have discussed are naturally meaningful in this variant of the cut game: for instance, an optimal state for $m=2$ is a configuration in which the weighted cut size is maximized.

\section{CONCLUSIONS AND FUTURE WORK}

In this paper, we have studied the dynamics of coalition formation under marginal contribution-based profit-division schemes. We have introduced three classes of noncooperative games that can be constructed from any cooperative game with a submodular utility function. We have shown that all three profit-distribution schemes considered in this paper have desirable properties: all three games admit a Nash equilibrium, and even the worst Nash equilibrium is within a factor of 2 from the optimal configuration. In addition, for fair-value games and labor union games, a natural dynamics process quickly converges to a state with a fairly high total profit. Thus, when rules for sharing the payoff are fixed in advance, we can expect a system composed of players who choose approximate best response strategies to quickly converge to an acceptable set of teams. 
We observe that our results have implications for combinatorial auctions with submodular bidders. Indeed, if we identify bidders and items with parties and players, respectively, then the social welfare in a combinatorial auction corresponds to the total profit in the respective profit-sharing game. Consequently, Corollaries 4.3 and 4.7 provide $(2+\epsilon)$-approximation algorithms to optimal social welfare in combinatorial auctions. These algorithms operate by starting with an arbitrary allocation of items to bidders and then letting the items migrate from one bidder to another for a polynomial number of steps according to artificially constructed "item utility functions." At each step, the next item to migrate and its final destination can be determined using two value queries. Thus, these algorithms run in polynomial time in the value-query model. While such algorithms are already known [28], improving over the approximation ratio of 2 in the value-query model appears to be challenging (but see [19]).

Of course, the picture given by our results is far from complete; rather, our work should be seen as a first step toward understanding the behavior of myopic selfish agents in coalition-formation settings. In particular, our results seem to suggest that keeping track of the history of the game and distributing payoffs in a way that respects players" "seniority" leads to better stability properties; it would be interesting to see whether this observation is true in practice, and whether it generalizes to other settings, such as congestion games.

In contrast to the previous work on cost-sharing and profit-sharing games, our work does not assume that the game's payoffs are given by an underlying combinatorial structure. Rather, our results hold for every cooperative game with a submodular utility function, and in particular, do not depend on whether it is compactly representable.

Further, all of our results are noncomputational in nature. Indeed, since the standard representation of cooperative games is exponential in the number of players, one can hope to obtain meaningful complexity results only for subclasses of cooperative games that possess a succinct representation; identifying such classes and proving complexity results for them is a promising research direction.

In our study of labor union games, we took a somewhat unusual modeling approach: we considered a system described by a sequence of states each of which induces a noncooperative game, and proved convergence results about the dynamics of such systems. This approach can be extended to other classes of games such as congestion games; indeed, there are real-life systems in which a player's payoff depends on who selected a certain resource before him. It would be interesting to see whether the known results for congestion games extend to this setting.

\section{ACKNOWLEDGMENTS}

A preliminary version of this work [5] has been published in the proceedings of the 22nd International Joint Conference on Artificial Intelligence (IJCAI 2011).

\section{FUNDING}

This work was supported by a NAP grant, NRF (Singapore) under grant NRF RF200908, and a STAR SINGA graduate fellowship. 


\section{REFERENCES}

[1] F. Abed and C. Huang. "Preemptive Coordination Mechanisms for Unrelated Machines." In ESA, pp. 12-23, 2012.

[2] H. Ackermann, H. Röglin, and B. Vöcking. "On the Impact of Combinatorial Structure on Congestion Games.” J. ACM 55:6 (2008), 25:1-25:22.

[3] S. Airiau and S. Sen. "A Fair Payoff Distribution for Myopic Rational Agents." In AAMAS, pp. 1305-1306, 2009.

[4] E. Anshelevich, A. Dasgupta, J. M. Kleinberg, É. Tardos, T. Wexler, and T. Roughgarden. "The Price of Stability for Network Design with Fair Cost Allocation.” SIAM J. Comput., 38:4 (2008), 1602-1623.

[5] J. Augustine, N. Chen, E. Elkind, A. Fanelli, N. Gravin, and D. Shiryaev. "Dynamics of ProfitSharing Games." In IJCAI, pp. 37-42, 2011.

[6] B. Awerbuch, Y. Azar, A. Epstein, V. S. Mirrokni, and A. Skopalik. "Fast Convergence to Nearly Optimal Solutions in Potential Games." In Proceedings of the ACM Conference on Electronic Commerce, pp. 264-273, 2008.

[7] Y. Azar, K. Jain, and V. S. Mirrokni. “(Almost) Optimal Coordination Mechanisms for Unrelated Machine Scheduling.” In SODA, pp. 323-332, 2008.

[8] A. Bhalgat, T. Chakraborty, and S. Khanna. "Approximating Pure Nash Equilibrium in Cut, Party Affiliation, and Satisfiability Games.” In EC, pp. 73-82, 2010.

[9] I. Caragiannis. "Efficient Coordination Mechanisms for Unrelated Machine Scheduling." Algorithmica 66:3 (2013), 512-540.

[10] G. Chalkiadakis and C. Boutilier. "Bayesian Reinforcement Learning for Coalition Formation under Uncertainty.” In AAMAS, pp. 1090-1097, 2004.

[11] K. Chatterjee, B. Dutta, and K. Sengupta. A Noncooperative Theory of Coalitional Bargaining. Review of Economic Studies 60:2 (1993), 463-477.

[12] H. Chen and T. Roughgarden. "Network Design with Weighted Players." Theory Computing Systems 45:2 (2009), 302-324.

[13] H. Chen, T. Roughgarden, and G. Valiant. "Designing Network Protocols for Good Equilibria." SIAM J. Comput. 39:5 (2010), 1799-1832.

[14] S. Chien and A. Sinclair. "Convergence to Approximate Nash Equilibria In Congestion Games." In SODA, pp. 169-178, 2007.

[15] G. Christodoulou, V. S. Mirrokni, and A. Sidiropoulos. "Convergence and Approximation in Potential Games.” In STACS, pp. 349-360, 2006.

[16] G. Christodoulou, K. Mehlhorn, and E. Pyrga. "Improving the Price of Anarchy for Selfish Routing via Coordination Mechanisms.” In ESA, pp. 119-130, 2011.

[17] X. Deng and C. H. Papadimitriou. "On the Complexity of Cooperative Solution Concepts." Mathematics of Operations Research 19:2 (1994), 257-266.

[18] T. Dieckmann and U. Schwalbe. "Dynamic Coalition Formation and the Core." Journal of Economic Behavior and Organization 49 (2002), 363-380.

[19] S. Dobzinski and M. Schapira. "An Improved Approximation Algorithm for Combinatorial Auctions with Submodular Bidders.” In SODA, pp. 1064-1073, 2006.

[20] A. Fabrikant, C. H. Papadimitriou, and K. Talwar. "The Complexity of Pure Nash Equilibria." In STOC, pp. 604-612, 2004.

[21] P. von Falkenhausen and T. Harks. "Optimal Cost Sharing Protocols for Scheduling Games." In EC, pp. 285-294, 2011.

[22] A. Fanelli and L. Moscardelli. "On Best Response Dynamics in Weighted Congestion Games with Polynomial Delays.” Distributed Computing 24:5 (2011), 245-254.

[23] A. Fanelli, M. Flammini, and L. Moscardelli. "The Speed of Convergence in Congestion Games under Best-Response Dynamics.” ACM Transactions on Algorithms 8:3 (2012), 25:1-25:15.

[24] M. Gairing and R. Savani. "Computing Stable Outcomes in Hedonic Games." In SAGT, pp. 174$185,2010$. 
[25] M. Goemans, V. S. Mirrokni, and A. Vetta. "Sink Equilibria and Convergence." In FOCS, pp. 142-154, 2005.

[26] D. S. Johnson, C. H. Papadimitriou, and M. Yannakakis. "How Easy Is Local Search?” Journal of Computer and System Sciences 37 (1988), 79-100.

[27] K. Kollias and T. Roughgarden. "Restoring Pure Equilibria to Weighted Congestion Games." In ICALP (2), pp. 539-551, 2011.

[28] B. Lehmann, D. J. Lehmann, and N. Nisan. "Combinatorial Auctions with Decreasing Marginal Utilities." Games and Economic Behavior 55:2 (2006), 270-296.

[29] J. R. Marden and A. Wierman. "Distributed Welfare Games." Operations Research 61 (2013), 155-168.

[30] V. S. Mirrokni and A. Vetta. "Convergence Issues in Competitive Games." In Approximation, Randomization, and Combinatorial Optimization: Algorithms and Techniques, Lecture Notes in Computer Science 3122, pp. 183-194, 2004.

[31] B. Moldovanu and E. Winter. "Order Independent Equilibria." Games and Economic Behavior 9:1 (1995), 21-34.

[32] D. Monderer and L. S. Shapley. "Potential Games." Games and Economic Behavior 14:1 (1996), 124-143.

[33] A. Okada. "A Noncooperative Coalitional Bargaining Game with Random Proposers." Games and Economic Behavior 16 (1996), 97-108.

[34] R. W. Rosenthal. "A Class of Games Possessing Pure-Strategy Nash Equilibria." International Journal of Game Theory 2 (1973), 65-67.

[35] T. Sandholm and V. Lesser. "Coalitions among Computationally Bounded Agents." Artif. Intell. 94 (1997) 99-137.

[36] A. A. Schäffer and M. Yannakakis. "Simple Local Search Problems That Are Hard to Solve." SIAM J. Comput. 20:1 (1991), 56-87.

[37] L. S. Shapley. "A Value for $n$-Person Games." Contributions to the Theory of Games 2 (1953), 307-317.

[38] O. Shehory and S. Kraus. "Feasible Formation of Coalitions among Autonomous Agents in Nonsuperadditve Environments." Comp. Intell. 15 (1999), 218-251.

[39] A. Skopalik and B. Vöcking. "Inapproximability of Pure Nash Equilibria." In STOC, pp. 355$364,2008$.

[40] T. Tscheuschner. "Settling the Complexity of Local Max-Cut (Almost) Completely." In ICALP, pp. 171-182, 2011.

[41] H. Yan. "Noncooperative Selection of the Core." International Journal of Game Theory 31:4 (2003), 527-540. 